Check for updates

Cite this: Chem. Sci., 2017, 8, 5969

\title{
X-ray circular dichroism signals: a unique probe of local molecular chirality $\dagger$
}

\author{
Yu Zhang, (D) $\dagger^{\star a}$ Jérémy R. Rouxel, $\star^{\star a}$ Jochen Autschbach, (D) *b Niranjan Govind*c \\ and Shaul Mukamel ${ }^{* a}$
}

Core-resonant circular dichroism (CD) signals are induced by molecular chirality and vanish for achiral molecules and racemic mixtures. The highly localized nature of core excitations makes them ideal probes of local chirality within molecules. Simulations of the circular dichroism spectra of several molecular families illustrate how these signals vary with the electronic coupling to substitution groups, the distance between the X-ray chromophore and the chiral center, geometry, and chemical structure. Clear insight into the molecular structure is obtained through analysis of the $\mathrm{X}$-ray CD spectra.

Received 27th March 2017 Accepted 23rd June 2017

DOI: $10.1039 / \mathrm{c} 7 \mathrm{sc} 01347 \mathrm{~g}$

rsc.li/chemical-science positions where molecules are preferably excited by a spatially sculpted chiral field. Understanding the local variation of XCD signals within a molecule helps to further refine this approach ${ }^{5}$ by placing the X-ray chromophore in an optimal location. To first order in the incoming field (the linear response function is $\chi^{(1)}$, in general one must include a multipolar expansion of the radiation/matter coupling at least to first order in the wavevector $\mathbf{k}$, i.e. include the magnetic dipole and the electric quadrupole transition moments. The CD signal, defined as the difference between the absorption of left and right polarized light, ${ }^{2,9}$ directly targets the chiral part of the response tensor and vanishes for achiral molecules.

There are a wide variety of options as to how to conduct a CD experiment in the X-ray regime. In the soft X-ray range, circularly polarized light from synchrotron sources has been used for the past few decades..$^{10}$ XCD signals can be acquired in the frequency domain where a continuous wave can be scanned to generate the CD signal. Alternatively, in the time domain, the free induction decay after an impulsive excitation is measured and Fourier transformed. Recent technological developments allow the use of free electron laser (FEL) based X-ray light ${ }^{11-16}$ and tabletop sources based on a high harmonic generation (HHG) process. ${ }^{17,18} \mathrm{X}$-ray CD experiments are more challenging than their optical counterparts because of the lack of good circularly polarized X-ray light sources. Intense circularly polarized X-ray beams became available using helical undulators in the 1990s, ${ }^{19,20}$ and then a series of XCD measurements on single crystals were reported. ${ }^{21-23}$ So far, most XCD experiments have been carried out on crystals. The main type of X-ray circular dichroism technique has been XMCD,,$^{7,24,25}$ in which centrosymmetry is broken by an external static magnetic field, revealing information on the atomic magnetic properties. All amino acids in living bodies except for glycine are chiral. XCD experiments at the $\mathrm{C}, \mathrm{N}$ and $\mathrm{O}$ K-edges on small amino-acids were performed in the 2000s. ${ }^{26,27}$ Polypeptide chains can give

\footnotetext{
aDepartment of Chemistry, University of California, Irvine, CA 92697, USA. E-mail: yuz10@uci.edu; jrouxel@uci.edu; smukamel@uci.edu

${ }^{b}$ Department of Chemistry, University at Buffalo, State University of New York, Buffalo, NY 14260, USA. E-mail: jochena@buffalo.edu

'Environmental Molecular Sciences Laboratory, Pacific Northwest National Laboratory, Richland, WA 99352, USA. E-mail: niri.govind@pnnl.gov

$\dagger$ Electronic supplementary information (ESI) available: Molecular coordinates, more relevant molecular orbital plots and detailed calculation results. See DOI: $10.1039 / \mathrm{c} 7 \mathrm{sc} 01347 \mathrm{~g}$

$\ddagger$ These authors contributed equally to this work.
} 
characteristic UV-vis CD spectra which contain information about their conformations. Optical CD signals have been widely used to study ligand-protein interactions and the secondary and tertiary structures of proteins. ${ }^{28}$ The time-resolved vibrational CD technique has made it possible to observe ultrafast molecular structural changes. ${ }^{29}$ However, the relation of XCD signals to the molecular geometry and to the chemical and electronic structure has not been thoroughly established.

Here, we report core-level time-dependent density functional theory (TDDFT) ${ }^{30-37}$ based XCD simulations of several series of chiral molecules where a distinct X-ray chromophore (chlorine atom) is attached at various positions. The XCD signals at the chlorine $\mathrm{L}_{2,3}$-edges $(\sim 200 \mathrm{eV})$ provide a highly localized probe of the chirality. The electronic effect on XCD signals is studied in the following systems: chlorophenylethanol (CPEO, chemical structures shown in Fig. 1) and hexahelicene molecules $n$ chlorohexahelicene ( $n \mathrm{CHHC}, n=1-8$, structures shown in Fig. 3), which are benchmarks for global chirality in molecules that do not have chiral centers. We further examine a family of 1-bromo- $n$-chloronona-2,4,6,8-tetraen-1-amine (BnCTA, $n=2$ 10, structures shown in Fig. 5) molecules in which the X-raychromophore-chiral-center distance is varied. Finally, we demonstrate how the XCD signals vary in slightly modified chemical structures of the chiral centers of two selected BnCTA molecules.

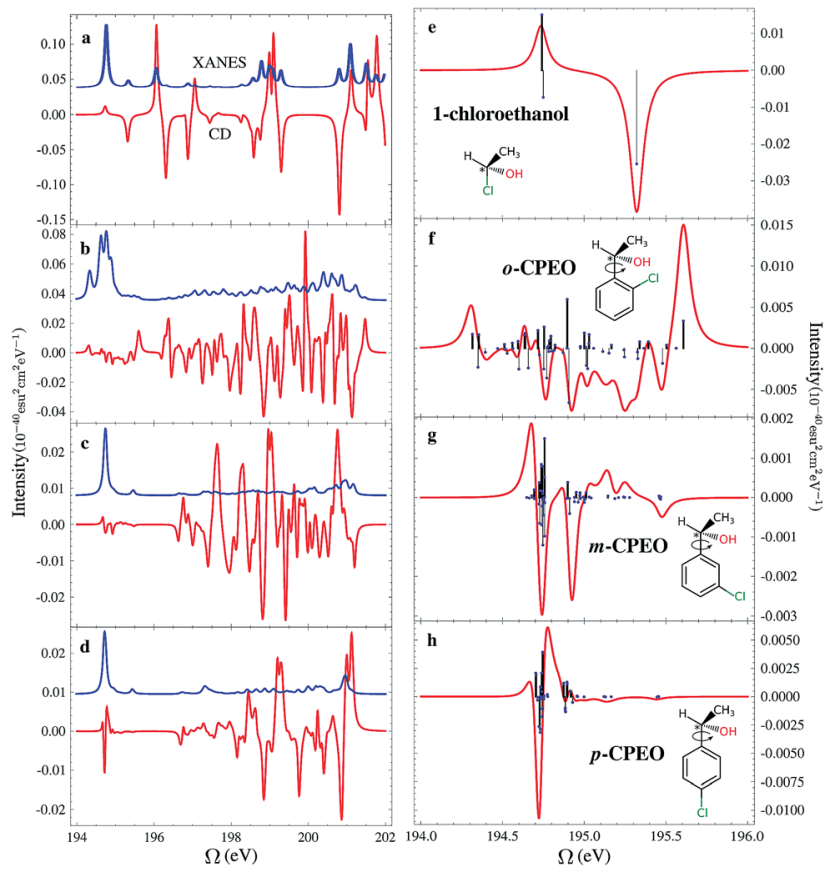

Fig. 1 Simulated XANES (blue) and rotationally averaged XCD (red) signals at the $\mathrm{Cl} \mathrm{L}_{2,3}$-edge on o,m,p-CPEO molecules. The chemical structures are shown in the right column with the chiral center marked by an asterisk. The corresponding XCD and XANES signals over a broad energy range are given in the left column. The right column shows the XCD spectra in the low-energy range. All XCD convoluted signals are plotted on the same scale and can be compared with each other directly. The XCD stick signals in the low-energy range are shown in the right column and are on arbitrary scales.
The paper is organized as follows: in section 2, we review the theory of the CD signals; computational details are provided in section 3; in section 4, we present and discuss the XCD simulation results; and finally we draw conclusions and outline some future directions in section 5 .

\section{The X-ray circular dichroism signal}

Circular dichroism (CD) is the simplest chiral optical technique widely used to measure enantiomeric excess. The CD signal is given by the difference in the absorption spectra of a left and right circularly polarized pulse. ${ }^{2}$

Our calculation starts with the Hamiltonian:

$$
H=H_{0}-\boldsymbol{\mu} \cdot \mathbf{E}(t)-\mathbf{m} \cdot \mathbf{B}(t)
$$

where $H_{0}$ represents the free molecule and the other terms represent the light-matter interaction, $\mathbf{E}(t)$ and $\mathbf{B}(t)$ are the electric and magnetic fields and $\boldsymbol{\mu}$ and $\mathbf{m}$ are the corresponding transition dipole moments, respectively. Specifically in isotropic randomly oriented ensembles the electric quadrupole moment $\mathbf{q}$ contribution to the CD signal averages to zero and is thus neglected. ${ }^{38}$ The absorption of a left (L) circularly polarized weak probe is given by:

$$
A^{\mathrm{L}}(\omega, \tau)=2 \omega \Im\left[\mathbf{E}^{\mathrm{L}^{*}}(\omega) \cdot \mathbf{P}^{\mathrm{L}}(\omega)+\mathbf{B}^{\mathrm{L}^{*}}(\omega) \cdot \mathbf{M}^{\mathrm{L}}(\omega)\right],
$$

where $\mathbf{P}$ is the electric polarization induced in the molecule, $\mathbf{M}^{\mathrm{L}}$ is the induced magnetization and $\mathfrak{\Im}$ denotes the imaginary part. The right circularly polarized signal is defined similarly using the superscript $\mathrm{R}$. The CD signal is given by:

$$
\begin{aligned}
& S_{\mathrm{XCD}}(\omega)=2 \omega \Im\left[\mathbf{E}^{\mathrm{L} *}(\omega) \cdot \mathbf{P}^{\mathrm{L}}(\omega)-\mathbf{E}^{\mathrm{R}^{*}}(\omega) \cdot \mathbf{P}^{\mathrm{R}}(\omega)+\mathbf{B}^{\mathrm{L} *}(\omega) \cdot \mathbf{M}^{\mathrm{L}}(\omega)\right. \\
& \left.-\mathbf{B}^{\mathrm{R} *}(\omega) \cdot \mathbf{M}^{\mathrm{R}}(\omega)\right] .
\end{aligned}
$$

Expanding the polarization and magnetization to first order in the incoming field and carrying out rotational averaging finally leads to: ${ }^{2}$

$$
S_{\mathrm{XCD}}(\omega)=\rho_{0} \frac{2}{3} \sum_{m} R_{m 0} g_{m 0}(\omega),
$$

where

$$
R_{m 0}=\Im[\boldsymbol{\mu} \cdot \mathbf{m}]
$$

is the rotatory strength ${ }^{2,39}$ of the 0 to $m$ transition, $\rho_{0}$ is the molecular density and $g_{m 0}(\omega)$ is a Lorentzian lineshape function centered at $\omega_{m 0}$ :

$$
g_{m 0}(\omega)=\frac{\Gamma_{m 0}}{\left(\omega-\omega_{m 0}\right)^{2}+\Gamma_{m 0}^{2}} .
$$

Here $\omega_{m 0}$ is the transition frequency from the ground state (0) to state $m$ and $\Gamma_{m 0}$ is the corresponding dephasing rate.

Eqn (4) can be measured directly in the frequency domain by using an incoming weak plane wave and scanning its wavelength. Alternatively, one can make use of ultrafast circularly polarized sources to measure XCD in the time domain. ${ }^{40}$ In this 
case, the signal is given by a free induction decay generated by the following polarization and magnetization:

$$
\begin{gathered}
\mathbf{P}^{(1)}(t)=\int_{0}^{+\infty} \mathrm{d} t_{1} \mathbf{R}_{\mathrm{em}}\left(t_{1}\right) \cdot \mathbf{E}\left(t-t_{1}\right) \\
\mathbf{M}^{(1)}(t)=\int_{0}^{+\infty} \mathrm{d} t_{1} \mathbf{R}_{\mathrm{me}}\left(t_{1}\right) \cdot \mathbf{B}\left(t-t_{1}\right)
\end{gathered}
$$

where the electric-magnetic response functions are given by:

$$
\begin{aligned}
& \mathbf{R}_{\mathrm{em}}\left(t_{1}\right)=\frac{i}{\hbar} \theta\left(t_{1}\right)\left\langle\left[\boldsymbol{\mu}\left(t_{1}\right), \quad \mathbf{m}(0)\right]\right\rangle \\
& \mathbf{R}_{\mathrm{me}}\left(t_{1}\right)=\frac{i}{\hbar} \theta\left(t_{1}\right)\left\langle\left[\mathbf{m}\left(t_{1}\right), \quad \boldsymbol{\mu}(0)\right]\right\rangle
\end{aligned}
$$

When the pulse is very short (impulsive), the incoming $\mathbf{E} / \mathbf{B}$ field can be approximated by a $\delta$ function and the emitted polarization and magnetization directly give the response function; no integrations are needed. After performing rotational averaging, the self-heterodyne frequency-dispersed linear absorption is finally given by:

$$
S_{\mathrm{XTRCD}}(\omega)=-4 \mathfrak{S}\left[\frac{1}{3} \operatorname{Tr} i\left(\mathbf{R}_{\mathrm{em}}(\omega)-\mathbf{R}_{\mathrm{me}}(\omega)\right)\right]
$$

\section{Computational approach}

Similarly to X-ray absorption, XCD signals of solids can be calculated in the framework of multiple scattering theory. ${ }^{41}$ Many program packages such as FEFF $^{42}$ and FDMNES $^{43}$ are available. The methods for calculating XCD signals in molecules have been summarized by Kimberg and Kosugi. ${ }^{\mathbf{4 4}}$ These methods include the static exchange (STEX), ${ }^{45-47}$ the random phase approximation (RPA) ${ }^{48}$ and the "Hartree-Fock excited state" methods.

In STEX, the occupied orbitals of a core-excited manyelectron system are approximated by the restricted open-shell Hartree-Fock (ROHF) converged occupied orbitals of the corresponding (N-1)-electron system. One core electron is then placed in an excited orbital, which is an eigenvector of the projected single-electron STEX Fock operator. ${ }^{46,47}$ This projection guarantees orthogonality between the occupied and excited orbitals. STEX can be combined with the transition potential method ${ }^{49,50}$ to study XCD signals. ${ }^{51}$ Other applications of STEX in XCD simulation can be found in ref. 52-54.

RPA, or time-dependent Hartree-Fock (TDHF), is a response theory in the frequency domain using the Hartree-Fock ground state orbitals. It was used to calculate the $\mathrm{C}, \mathrm{N}$ and $\mathrm{O}$ K-edge XCD signals of small molecules by Alagna et al. ${ }^{55}$ and was recommended by Kimberg and Kosgui ${ }^{44}$ as the most consistent and fastest method among the three.

The "Hartree-Fock excited state" method calculates each core-excited state through a self-consistent Hartree-Fock iteration. The obtained excited state orbitals should lie between the unrelaxed and relaxed STEX orbitals, and thus may provide better results than STEX does. However, solving the excited states one at a time is tedious and this also results in nonorthogonality between the excited states.

Another popular method for XCD simulation is the complex polarization propagator (CPP) method. ${ }^{56-58} \mathrm{CPP}$ is a response theory in which the complex electric-dipole polarizability tensor and the mixed electric dipole-magnetic dipole tensor of the system are solved from a linear response matrix equation under external field perturbations. CPP has been used by Norman and co-workers to simulate XCD spectra of many chiral molecules from small amino acids to fullerene. ${ }^{59-61}$ Generally in the CPP, ${ }^{62}$ damped response ${ }^{63,64}$ and real-time propagation ${ }^{65-68}$ approaches, explicit solving of the excited states is avoided, which facilitates calculations on systems with a high density of states. However, the fact that there is no direct access to the involved excited states also makes signal interpretation less straightforward.

Here, all XCD signals at the $\mathrm{Cl} \mathrm{L}_{2,3}$-edge were calculated using the CD spectrum module ${ }^{69-71}$ and the restricted excitation window TDDFT (REW-TDDFT) ${ }^{36,37}$ approach, implemented in the quantum chemistry package NWChem ${ }^{72}$ under the TammDancoff approximation (TDA). ${ }^{73-75}$ The rotatory strengths were calculated using the dipole-length formalism. The B3LYP hybrid functional ${ }^{76,77}$ was used. The cc-pVTZ ${ }^{78-80}$ basis set was used for all molecules except for the helicenes, where we used the basis set $6-311 \mathrm{G}^{* *}{ }^{\mathbf{8 1}, \mathbf{8 2}}$ We chose TDDFT as the method for XCD simulation for its balance between computational accuracy and cost. In addition, TDDFT is well developed, and large scale calculations can be started without too many trial-and-error tests.

We studied the $\mathrm{Cl} \mathrm{L}_{2,3}$-edge at $\sim 200 \mathrm{eV}$ rather than the $\mathrm{K}$ edge because the corresponding $2 \mathrm{p}$ core excitations are not as localized as the $1 \mathrm{~s}$ core excitations, hence the $\mathrm{L}_{2,3}$-edge XCD signals contain additional structural information on the entire molecule. Spin-orbit coupling (SOC) of the 2 p electrons splits the XANES spectra and the electron-hole exchange interaction blurs the physical picture of the independent particle, thus the experimental intensity branching ratio of the transitions from the $2 \mathrm{p}_{3 / 2}$ and $2 \mathrm{p}_{1 / 2}$ orbitals may stray far from the ideal value of $2: 1 .^{83}$ Recently a damped response study showed that this branching ratio in L-edge XANES was reproduced well from four-component full relativistic density function theory. ${ }^{\mathbf{8 4}}$ Similarly, SOC should also play an important role in L-edge XCD. However, the sizes of the molecules in this study and the numbers of the involved excited states prohibit a full relativistic calculation. Thus we have neglected SOC in our current study. We do not expect quantitative accuracy with the current calculations. However, there is every reason to expect that SOC should not affect the most important findings, i.e., for molecules with a chiral center the amplitude of the XCD signals strongly decreases with the distance between the X-ray chromophore and the chiral center if the corresponding excitations are very localized to the excited atom, and that for globally chiral molecules the XCD signals also strongly depend on the chromophore position. A two-component TDDFT approach is under development for future XCD simulations of large molecules.

Here we focus on the XCD signal patterns and report the raw core excitation energies. These calculations must be shifted to 
reproduce experimental core excitation energies. To eliminate numerical errors, we adopted a tight SCF convergence criterion (energy change of SCF cycles $<10^{-8}$ a.u.) and an ultrafine grid in all DFT calculations. Test calculations on achiral molecules which are similar to our studied molecules show that the calculated rotatory strengths are zero within the numerical accuracy of the calculation (at least 4 orders of magnitude smaller than the rotatory strengths for chiral molecules), which guarantees that the numerical errors are negligible. All impulsive signals are convoluted using a Lorentzian lineshape with a $0.1 \mathrm{eV}$ width, corresponding to the lifetime broadening of $\mathrm{Cl}$ $\mathrm{L}_{2,3}$ excitations. $^{85}$

XCD signals were found to be particularly sensitive to the local geometry of the excited atom. ${ }^{61}$ Here, we study molecules with slightly different chemical structures. Geometry optimization would make the local geometry of the excited atoms in those molecules different, so direct comparison of the XCD signals might be an issue. In order to cleanly isolate the effects from different substituent positions relative to the chiral centers from local structural changes that may occur upon optimization, the molecules were constructed from pre-defined molecular fragments. All geometries used in the calculations are provided in the ESI. $\dagger$

\section{Results and discussion}

\subsection{The chlorophenylethanol (CPEO) series}

The chemical structures of the CPEOs are shown in the right column of Fig. 1. The chlorine X-ray chromophore is positioned at the ortho-, meta-, or para-position. The XCD signal of 1chloroethanol is also provided for comparison. The XCD and Xray absorption near edge structure (XANES) signals in the simulated energy range (194-202 eV) are shown in the left column of Fig. 1. The right column shows the XCD signals on an expanded scale in the low-energy range (194-196 eV) around the main XANES resonance. Stick spectra of the XCD signals in this energy range are also provided.

We have held the geometry of the chiral center and the substituted benzene ring fixed, and only allowed the $\mathrm{C}-\mathrm{C}$ single bond to rotate. We chose a series of geometries with $\mathrm{C} 1-\mathrm{C} 2-\mathrm{C} 3-$ $\mathrm{C} 4$ torsion angles of 0, 60, 120, 180, 240 and 300 degrees. Here $\mathrm{C} 1$ denotes the $\mathrm{C}$ atom bound to $\mathrm{Cl}$ or $\mathrm{H}$ at the $o$-position; $\mathrm{C} 2-\mathrm{C} 3$ is the single bond connecting the chiral center and the benzene ring; and $\mathrm{C} 4$ is the $\mathrm{C}$ atom in $-\mathrm{CH}_{3}$. The XCD signals calculated for these geometries were used to compute the averaged signals. Averaging is necessary because the $\mathrm{C}-\mathrm{C}$ single bond can rotate freely at room temperature. We can see in Fig. 1 that the XCD signals of 1-chloroethanol where the $\mathrm{Cl}$ atom is directly bound to the chiral center are much stronger than those of the CPEOs.

The rotationally averaged XANES spectra of $m$ - and $p$-CPEO are similar and show a strong peak below $195 \mathrm{eV}$ and many weak features in the high-energy range. The corresponding spectrum for $o$-CPEO has a richer peak pattern. This is because of the close proximity of the $\mathrm{Cl}$ atom to the chiral center, so that the rotation of the chiral center may shift the strong core excitation energies. Only limited molecular structural information can be inferred from the XANES spectra while the XCD spectra are more informative.

The XCD signal intensities of the CPEOs are summarized in Table 1. We have examined the integrated absolute value intensity in various energy regimes from low to high, as well as the peaks with the largest intensities in these energy regimes. Representative particle molecular orbitals (MOs) indicate that the low energy excitations are localized to the $\mathrm{C}-\mathrm{Cl}$ bond and the benzene ring (see Fig. 2), without a substantial contribution from the atomic orbitals of the chiral center. The high-energy excitation MOs are delocalized to the chiral center (see Fig. 2). This explains why the XCD signals are weak in the low-energy regime and become stronger at higher energies. For low energy excitations, the magnitudes of the XCD integrated intensities of different isomers are ordered as: $p \sim o>m$. Dispersed XCD signals in the low energy regime (right column of Fig. 1) suggest the XCD intensity order $o>p \gg m$, supporting the common chemical intuition on the electronic coupling strength order of different substitution positions on the benzene ring. ${ }^{86}$ The $\mathrm{C}-\mathrm{C}$ single bond rotation does not considerably change the energies but can flip the sign of the XCD signal for the same core excitation. For $m$-CPEO, signals with opposite signs appear at very close energies, which means that the $\mathrm{C}-\mathrm{C}$ bond rotation does not shift the core excitations too much, while for the $o$ and $p$ isomers the $\mathrm{C}-\mathrm{C}$ bond rotation shifts the excitation energies more and makes the excitations more distinct. So the cancellation of the XCD signals with opposite signs is not that serious in $o$ and $p$-CPEO, compared to that in $m$-CPEO, which makes the XCD intensities of $o$ and $p$ CPEO much larger than that of $m$-CPEO (see the vertical axis scales of panels (f) and (g) in Fig. 1). Again, it indicates that $m$ CPEO has the weakest electronic coupling between the $\mathrm{Cl}$ atom and the chiral center. Low energy core excitations are localized and the corresponding XCD signals can detect electronic effects at different substituent positions.

For other higher energy regimes, taking the integrated and strongest peak intensities together, we find the XCD intensity to vary as $o>m>p$. This is because the orbitals involved in the high energy excitations are more delocalized than their low energy counterparts, and so the electronic effects of different substitution positions become less important and the corresponding XCD signals are determined by other factors such as the chiral-center-chromophore distance.

\subsection{The $n$-chlorohexahelicene (nCHHC) series}

Helicenes are well-studied globally chiral molecules. ${ }^{87}$ The chirality stems from their screw-like geometry rather than a local chiral center. We have studied a family of chlorine substituted hexahelicenes. If the excitations are local, the corresponding XCD signals should be weak since they don't probe the global structure. We expect excitations with MOs delocalized over the whole molecule to have strong XCD activity. Note that the chiral signals further depend on the magnitudes of the corresponding electric and magnetic transition dipoles.

The molecular structures and the corresponding XANES and XCD signals are depicted in Fig. 3 for the right-handed (P)- 
Table 1 XCD signal summary of CPEOs

\begin{tabular}{|c|c|c|c|c|c|c|}
\hline \multirow{2}{*}{$\begin{array}{l}\text { Energy regime } \\
(\mathrm{eV})\end{array}$} & \multicolumn{3}{|c|}{$\begin{array}{l}\text { Integrated absolute intensity }\left(10^{-40} \mathrm{esu}^{2}\right. \\
\left.\mathrm{cm}^{2}\right)\end{array}$} & \multicolumn{3}{|c|}{ Strongest peak/intensity $\left(\mathrm{eV} / 10^{-40} \mathrm{esu}^{2} \mathrm{~cm}^{2}\right)$} \\
\hline & $o$-CPEO & $m$-СРEO & $p$-СРEO & $o$-СРEO & $m$-СРEO & $p$-CPEO \\
\hline $194-196$ & 0.05042 & 0.04196 & 0.05420 & $194.91 /-0.02990$ & $194.76 / 0.03286$ & $194.75 / 0.02883$ \\
\hline $196-198$ & 0.1170 & 0.03514 & 0.01651 & $197.30 /-0.04449$ & $197.66 / 0.01306$ & $196.76 / 0.005288$ \\
\hline $198-200$ & 0.2664 & 0.1767 & 0.09356 & 199.91/0.1926 & 199.76/0.03341 & $199.20 / 0.02534$ \\
\hline
\end{tabular}

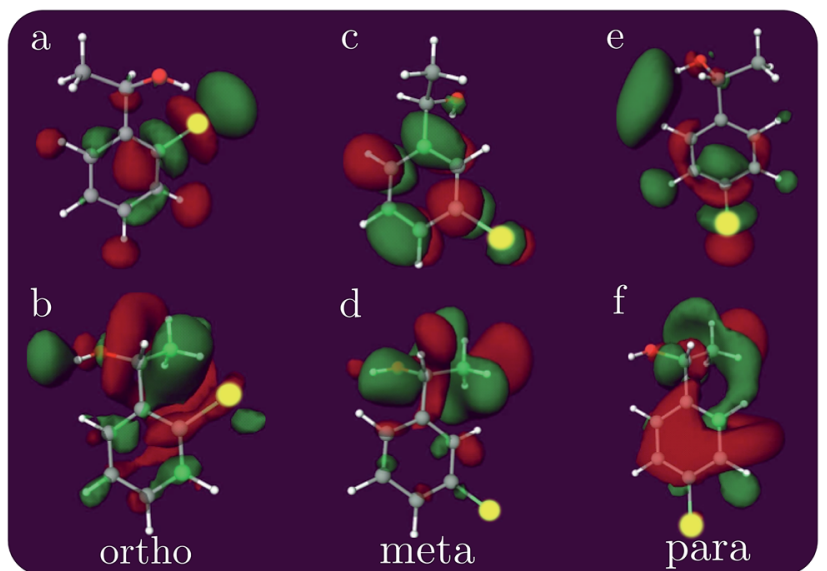

Fig. 2 Representative particle MOs of the $\mathrm{Cl} 2 \mathrm{p}$ core excitations of ortho, meta and para CPEOs. The X-ray chromophore is highlighted in yellow. The energies of the excited states and the $\mathrm{Cl}$ coefficients of the displayed MOs are (a) $194.90 \mathrm{eV},-0.95$; (b) $199.90 \mathrm{eV}, 0.88$; (c) $194.76 \mathrm{eV}, 0.68$; (d) $200.72 \mathrm{eV}, 0.58$; (e) $194.75 \mathrm{eV},-0.58$; and (f) $200.88 \mathrm{eV}, 0.60$. Additional relevant MOs are presented in the ESI. $\dagger$

hexahelicene enantiomer (CD signals for the left-handed (M) enantiomer have the opposite sign). The XCD signals vary strongly between the 8 possible Cl-substitution sites. The XANES signals of the $n$ CHHCs do not differ appreciably until the energy becomes very high $(>201 \mathrm{eV})$. Interestingly, lowenergy excitations lead to significant XCD intensities only for 1CHHC (panel (a) of Fig. 3); all other molecules have strong XCD features at energies greater than $198 \mathrm{eV}$. This is confirmed by displaying the relevant MOs. Typical MOs of low- and highenergy excitations of 1CHHC and 5CHHC are shown in Fig. 4. The particle MOs of low-energy excitations in all $n$ CHHCs are localized to the $\mathrm{C}-\mathrm{Cl}$ bond (e.g., panel (c) in Fig. 4) except for $1 \mathrm{CHHC}$, in which they are not localized. This explains why only $1 \mathrm{CHHC}$ has strong XCD features in the low energy regime. In contrast, high-energy excitations usually involve MOs delocalized over the entire molecule (e.g., panel (d) in Fig. 4), leading to strong XCD signals.

The rotatory strength, eqn (5), is given by the scalar product of the electric and magnetic transition dipoles which depends on the dipole magnitudes and their relative angle. Hence, the involvement of delocalized particle MOs is only a necessary but not a sufficient condition for a strong XCD feature. The calculated magnitudes of the electric and magnetic transition dipoles of all the studied $n$ CHHCs as well as the angles between the two dipoles are listed in the ESI. $\dagger$ In general, high-energy $\mathrm{Cl} 2 \mathrm{p}$ excitations in the $n$ CHHCs are delocalized and XCD can be used to provide structural information on molecules with global chirality.

\subsection{The 1-bromo-n-chloronona-2,4,6,8-tetraen-1-amine (BnCTA) series}

In the BnCTA molecules, a local chiral center is located at the tip of a long conjugated $\mathrm{C}-\mathrm{H}$ double bond chain. The chromophore-chiral-center distance can be varied by moving the $\mathrm{Cl}$ substituent along the chain. Fig. 5 depicts the XANES and XCD signals. One may expect the latter to decrease with the chromophore-chiral-center distance. Indeed, the intensities in the low-energy regimes show a roughly decaying behavior with the Cl-chiral-center distance, as shown by the plots of both the individual and integrated XCD signal intensities (see Fig. 6). B2CTA, in which the $\mathrm{Cl}$ atom is the closest to the chiral center in

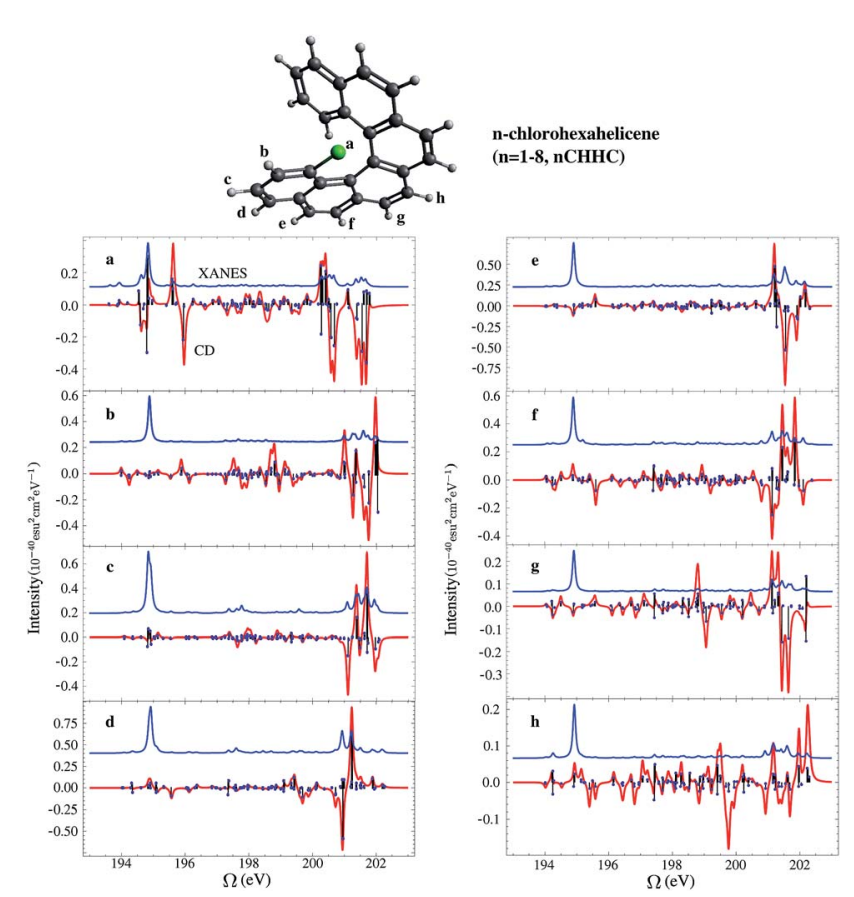

Fig. 3 Simulated XCD (red) and XANES (blue) signals at the $\mathrm{Cl} \mathrm{L}_{2,3}$ edge of $n$-chlorohexahelicene $(n \mathrm{CHHC}$ ) molecules. Panels (a to $\mathrm{h}$ ) correspond to the $n \mathrm{CHHC}$ molecules with the chlorine atoms placed at different positions as indicated by the letter labels on the molecule. XCD stick signals are shown on arbitrary scales. 


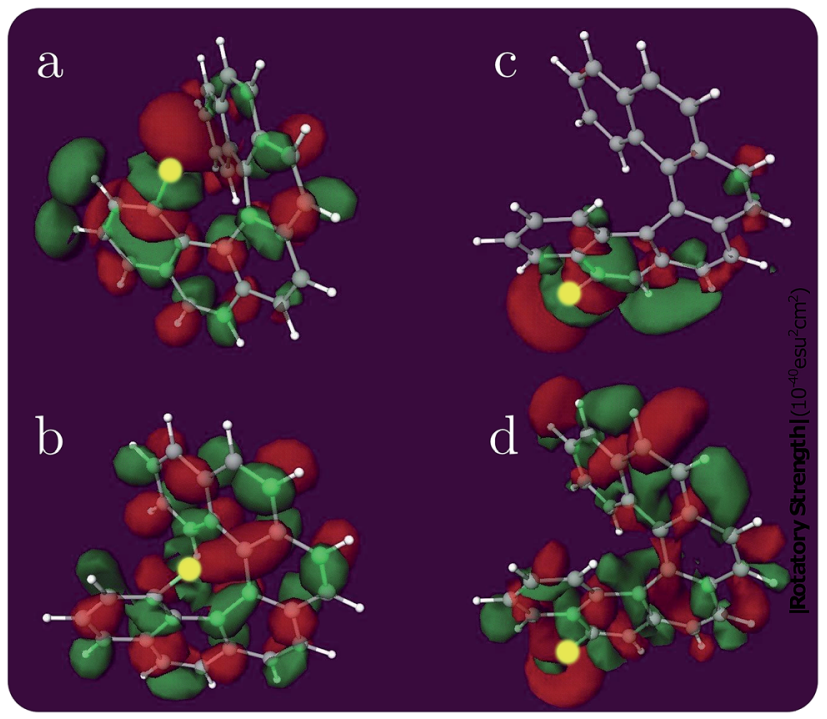

Fig. 4 Representative particle MOs of the $\mathrm{Cl} 2 \mathrm{p}$ core excitations of selected $n \mathrm{CHHCs}$ at low and high energy excitation. The X-ray chromophore is highlighted in yellow. The excitation energy and the Cl-type coefficient are, respectively: (a) $194.83 \mathrm{eV}, 0.55$; (b) $194.83 \mathrm{eV}$, 0.50; (c) $194.89 \mathrm{eV},-0.92$; and (d) $201.53 \mathrm{eV},-0.59$. Additional relevant MOs are presented in the ESI. $\dagger$
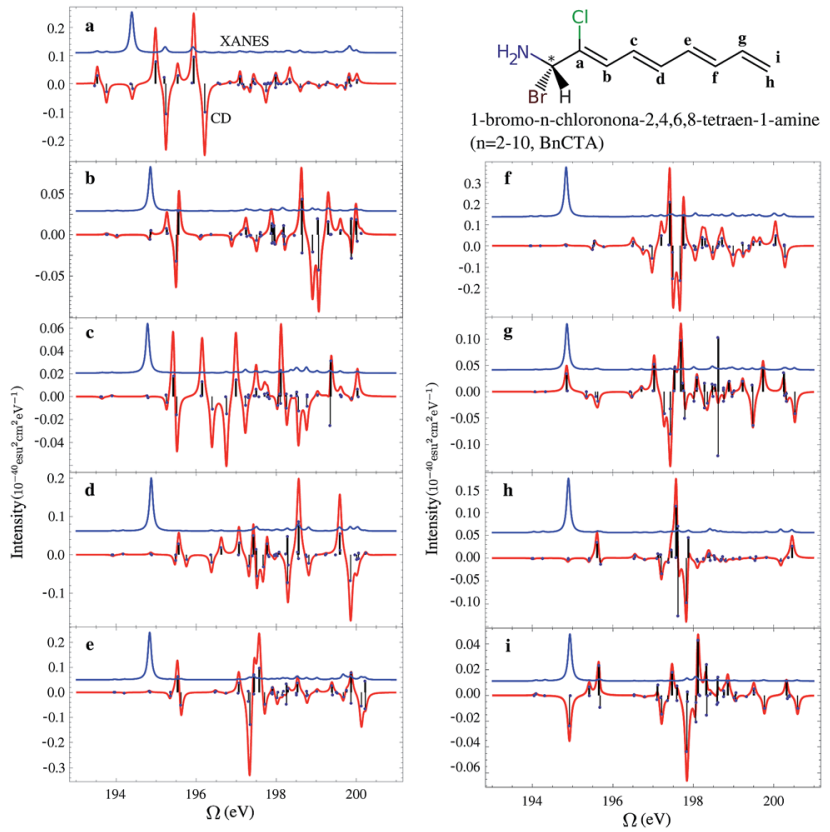

Fig. 5 Simulated X-ray CD (red) and XANES (blue) signals at the $\mathrm{Cl}_{2,3^{-}}$ edge of various 1-bromo- $n$-chloronona-2,4,6,8-tetraen-1-amine (BnCTA) molecules. Panels (a to i) correspond to different $\mathrm{X}$-ray chromophore locations as indicated in the Lewis structure (top right). XCD stick signals are shown on arbitrary scales.

the studied series, has much stronger XCD signals in the lowenergy regimes (193-195 and 195-197 eV). The other BnCTAs exhibit much weaker signals in these energy regimes, and the integrated XCD intensities only show small fluctuations as the

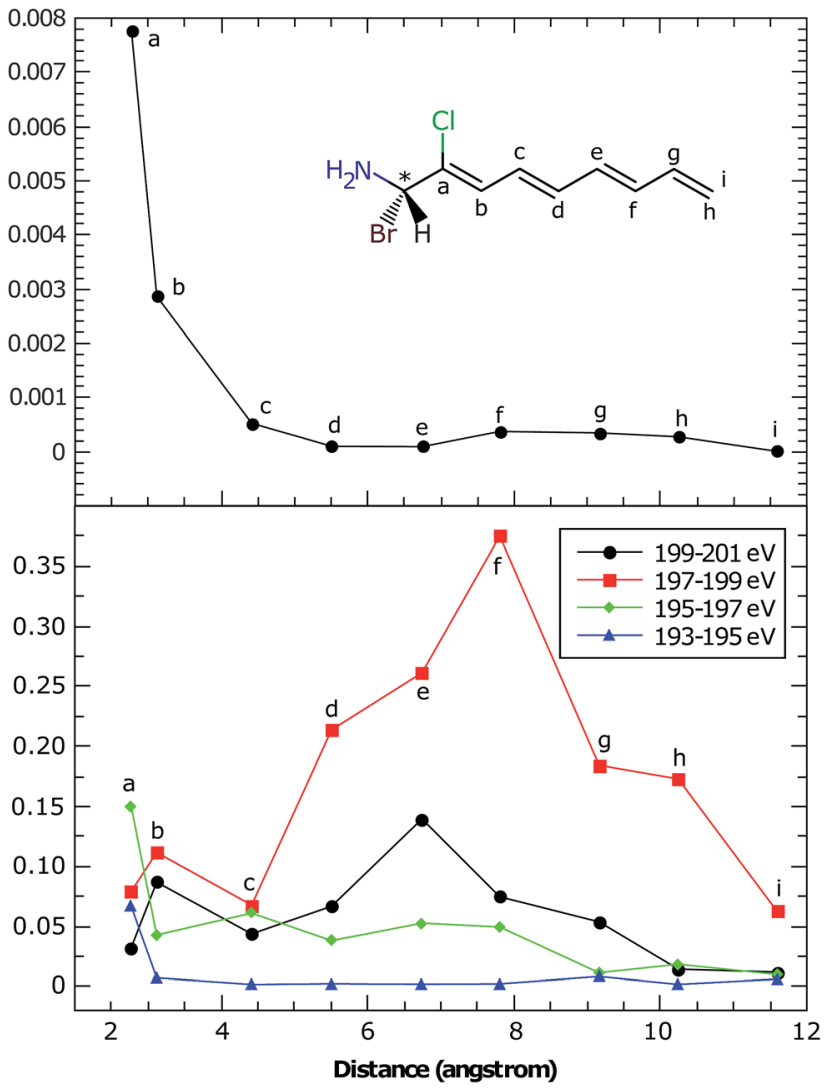

Fig. 6 Top: magnitude of the rotatory strength as a function of the distance between the X-ray chromophore and the chiral center at the one of the strongest XAS peaks: (a) $194.40 \mathrm{eV}$; (b) $194.86 \mathrm{eV}$; (c) $194.79 \mathrm{eV}$; (d) $194.88 \mathrm{eV}$; (e) $194.84 \mathrm{eV}$; (f) $194.85 \mathrm{eV}$; (g) $194.86 \mathrm{eV}$; (h) $194.90 \mathrm{eV}$; and (i) $194.93 \mathrm{eV}$. Bottom: magnitude of the rotatory strength as a function of the distance between the X-ray chromophore and the chiral center integrated over various energy windows for the BnCTAs.

Cl-chiral-center distance increases. Again, this implies that the low-energy excitations are localized near the $\mathrm{Cl}$ atom. The single particle picture of an excitation becomes more ambiguous when multiple orbital pairs are involved. We have used the collective electronic oscillator (CEO ${ }^{86}$ plots of a transition density matrix (TDM) to analyze the character of the corresponding excitations. These plots provide a real-space representation of the absolute values of the transition density matrix elements coarse-grained to various atoms (represented by indices in the plot). CEO plots of representative low energies of B2CTA ( $\mathrm{Cl}$ is the closest to the chiral center in the series) and B10CTA ( $\mathrm{Cl}$ is the farthest to the chiral center in the series) are shown in Fig. 7(a) and (b), respectively. We clearly see that the low-energy excitations are localized near the $\mathrm{Cl}$ atom and B2CTA has weak transitions between the $\mathrm{Cl}$ atom (index 8) and the chiral center (indices 35), while in B10CTA the $\mathrm{Cl}$ atom is too far away to affect the chiral center. This explains why B2CTA has a stronger XCD activity in the low-energy regime.

Fig. $7(\mathrm{c})$ shows that higher energy excitations are more delocalized along the entire chain. XCD signals at higher energies are complicated (see Fig. 5 and 6 bottom panel). Some 

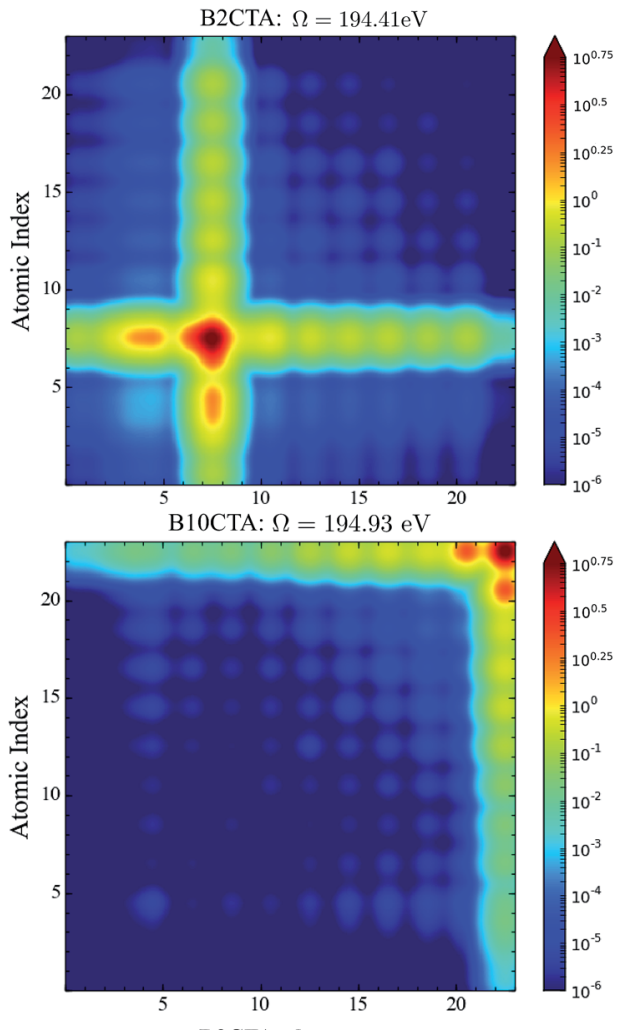

B2CTA: $\Omega=199.83 \mathrm{eV}$

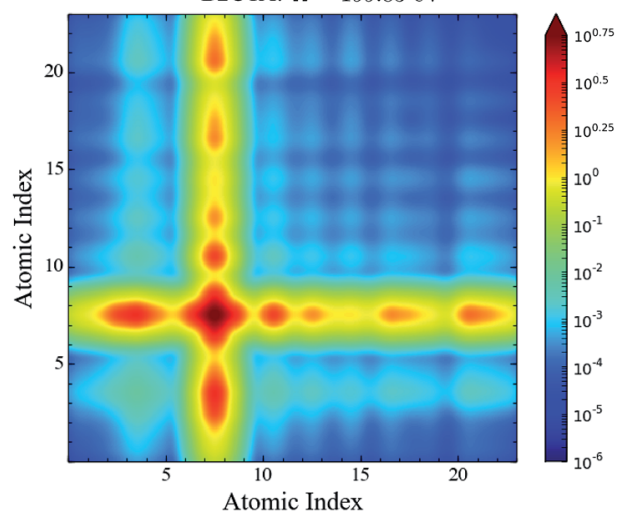

Fig. 7 CEO plots ${ }^{86}$ of the excitation at $194.41 \mathrm{eV}$ of B2CTA (top panel); the excitation at $194.93 \mathrm{eV}$ of B10CTA (middle panel); and the excitation at $199.83 \mathrm{eV}$ of B2CTA (bottom panel). In the top and bottom panels, the $\mathrm{Cl}$ atomic index is 8 , and in the middle panel the $\mathrm{Cl}$ atomic index is 23. In all plots the chiral center atomic indices are 1-5 and all the other atomic indices are for the conjugated long chain.

excitations are delocalized, but have a charge-transfer-like character, which leads to very small electric transition dipoles and XCD signals (e.g., B10CTA at $200.32 \mathrm{eV}$ ). B9CTA has weak XCD signals in the high energy regime because of the small angles between the electric and magnetic transition dipoles (e.g. $89.85^{\circ}$ for B9CTA at $200.46 \mathrm{eV}$ ). It is then difficult to interpret XCD intensities in the high-energy regimes in a simple manner. MO or CEO analysis only offer information about the transition dipole magnitudes but no direct information about the angles between those dipoles, which are determined by the molecular configuration and affect the rotatory strength. The magnitudes of the electric and magnetic transition dipoles of all BnCTAs studied as well as the angles between the two dipoles are provided in the ESI. $\dagger$ In summary, XCD signals in different energy regimes provide a fine description of the electronic coupling between the X-ray chromophore and the chiral center as well as clear structural information on the relative positions of the chiral center and the X-ray chromophore.

We conclude the study of this molecular series by examining the influence of different types of chiral centers. When the chiral center is changed, e.g. one $-\mathrm{H}$ is replaced by $-\mathrm{CH}_{3}$ in the BnCTA molecules, the XCD spectra are altered. We have modified the chiral centers in B3CTA and B4CTA as described and calculated the corresponding XCD spectra as displayed in Fig. 8. As before, the XANES spectra of all the species are very similar. The calculated XCD spectra of the original BnCTA and their modified counterparts are generally similar in shape too. One may find that peaks with similar characters shift slightly. To evaluate the effect of the chiral center variation on the XCD signals, we labeled seven major strong peaks of each species in the simulated spectra (see Fig. 8) and calculated their intensity differences. The results are shown in Table 2. Under the chiral center change, three major peaks ( $\mathrm{A} 2 / \mathrm{A}^{\prime}, \mathrm{A} 3 / \mathrm{A}^{\prime}$ and $\left.\mathrm{A} 4 / \mathrm{A} 4^{\prime}\right)$ in the energy regime of 195-199 eV have significant intensity variations, while only the peak around $197 \mathrm{eV}\left(\mathrm{B} 6 / \mathrm{B6}^{\prime}\right)$ changes in the higher energy regime. The intensity changes of other peak pairs are very small. This comparison of B3CTA, B4CTA and their modified forms indicates that B4CTA and its modified form show more differences in the XCD spectra, which is supported by the chemical structure analysis. In B3CTA the $\mathrm{Cl}$ atom is located at the cis-position of the changed atom, while in $\mathrm{B} 4 \mathrm{CTA}$ the $\mathrm{Cl}$ atom is at the trans-position. The cis-species are more sensitive to changes in the chiral center. This

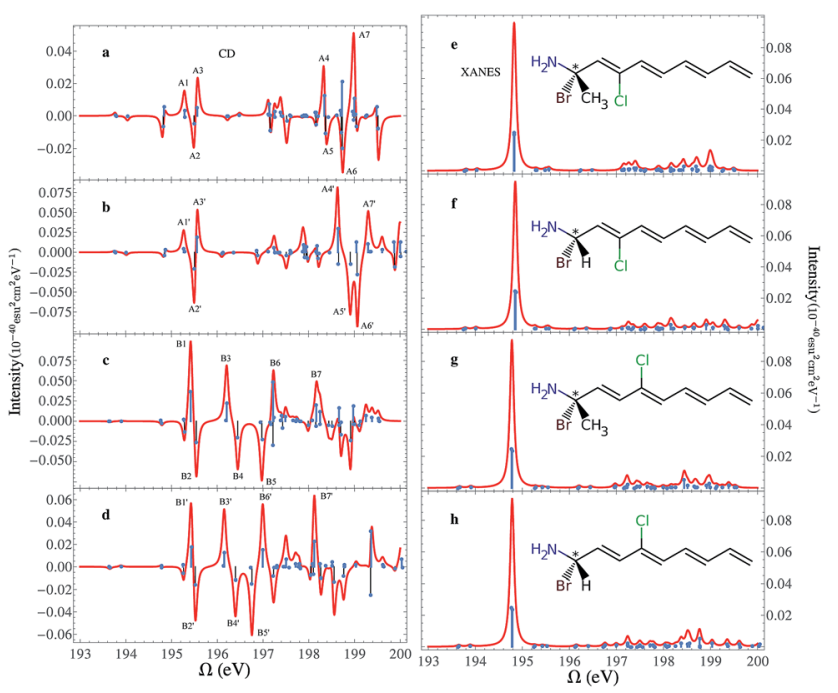

Fig. 8 Comparison of the simulated $\mathrm{Cl} \mathrm{L}_{2,3}$-edge $\mathrm{XCD}$ signals of two selected BnCTA molecules with those of the corresponding molecules with slightly different chiral centers. The left column shows the XCD signals. The corresponding simulated XANES signals and chemical structures are given in the right column. Stick signals are plotted on arbitrary scales. 
Table 2 XCD intensity differences of certain peaks under chiral center modification

\begin{tabular}{lc}
\hline & $\begin{array}{l}\text { Intensity difference } \\
\left(10^{-42} \mathrm{esu}^{2} \mathrm{~cm}^{2}\right)\end{array}$ \\
\hline Peak pairs & -0.08275 \\
$\mathrm{~A} 1-\mathrm{A} 1^{\prime}$ & 1.232 \\
$\mathrm{~A} 2-\mathrm{A} 2^{\prime}$ & -1.058 \\
$\mathrm{~A} 3-\mathrm{A} 3^{\prime}$ & -1.122 \\
$\mathrm{~A} 4-\mathrm{A} 4^{\prime}$ & 0.1062 \\
$\mathrm{~A} 5-\mathrm{A} 5^{\prime}$ & 0.3182 \\
$\mathrm{~A} 6-\mathrm{A} 6^{\prime}$ & 0.2312 \\
$\mathrm{~A} 7-\mathrm{A} 7^{\prime}$ & 0.6503 \\
$\mathrm{~B} 1-\mathrm{B} 1^{\prime}$ & -0.3057 \\
$\mathrm{~B} 2-\mathrm{B} 2^{\prime}$ & 0.3011 \\
$\mathrm{~B} 3-\mathrm{B} 3^{\prime}$ & -0.2624 \\
$\mathrm{~B} 4-\mathrm{B} 4^{\prime}$ & -0.1442 \\
$\mathrm{~B} 5-\mathrm{B} 5^{\prime}$ & 1.406 \\
$\mathrm{~B} 6-\mathrm{B} 6^{\prime}$ & -0.4992 \\
$\mathrm{~B} 7-\mathrm{B} 7^{\prime}$ &
\end{tabular}

demonstrates that XCD signals can sensitively probe the relative configuration of chemical groups.

\section{Conclusions and future perspectives}

We have investigated the local chirality in several families of molecules. We first considered the role of ortho, meta and para substitution in CPEO molecules and its interaction with the chiral center. We then discussed the localization of the interactions in hexahelicene for low and high energy core transitions, and also studied the decay of the chiral signal with the distance of the X-ray chromophore from the chiral center in linear BnCTA molecules. We have further shown that different chiral centers have different XCD signatures depending on the relative structures of the chemical groups.

The simulation of the XCD spectra of molecules is challenging. The relevant quantity is the rotatory strength, whose magnitude depends strongly on the angle between the electric and the magnetic dipoles. This angle is sensitive to the approximations made in the computational model. In addition, XCD signals are very sensitive to the local geometry in the vicinity of the absorbing center. Simulating the XCD spectra of highly flexible molecules is far more challenging and demanding due to conformational changes and vibrational effects and their impact on rotatory strengths resulting from closely spaced states. Even though TDDFT is, in principle, an exact theory and provides a computationally affordable framework to calculate XCD spectra, as we have demonstrated in this paper, the approximate nature of the exchange-correlation functionals leaves much room for improvement and for more systematic studies of molecules in realistic dynamical environments.

The development of new sources of X-ray circularly polarized light allows us to investigate the spectroscopic and structural properties of molecules. Circular dichroism is the simplest experimental probe of chirality and is now readily achievable by various X-ray sources. For molecules with a chiral center, XCD simulations indicate that the amplitude of the signals strongly decreases with the distance between the X-ray chromophore and the chiral center if the corresponding excitations are very localized to the excited atom. For globally chiral molecules, experimental XCD signals also strongly depend on the chromophore position because of different local chemical environments. Optical CD signals have been routinely used to detect the absolute configuration of large molecules for a long time. XCD signals can reveal more detailed information about the relative configuration of different chemical groups in a molecule, thanks to the localization of the core excitations invoked by Xrays.

With the advancement of X-ray laser technology, the challenges of XCD experiments will be overcome and more experiments on complicated molecular systems such as proteins are expected. The combination of XCD with time-domain techniques such as time-resolved $\mathrm{CD}^{88}$ provides a natural link between structural and functional properties. Transient XCD spectroscopy can be further used to monitor asymmetric chemical reactions.

\section{Acknowledgements}

The support of the National Science Foundation (grant CHE1361516) and the Chemical Sciences, Geosciences, and Biosciences division, Office of Basic Energy Sciences, Office of Science, U.S. Department of Energy through Awards No. DEFG02-04ER15571 (S. M.) and KC030105066418 (N. G.) is gratefully acknowledged. J. R was supported by the DOE grant. J. A. acknowledges the National Science Foundation (CHE-1560881) for financial support. A portion of the research was performed using EMSL, a DOE Office of Science User Facility sponsored by the Office of Biological and Environmental Research and located at PNNL. PNNL is operated by Battelle Memorial Institute for the United States Department of Energy under DOE contract number DE-AC05-76RL1830.

\section{References}

1 M. Quack, J. Stohner and M. Willeke, Annu. Rev. Phys. Chem., 2008, 59, 741-769.

2 Circular Dichroism, Principles and Applications, ed. N. Berova, K. Nakanashi and R. W. Woody, Wiley-VCH, Inc., 2nd edn, 2000.

3 A. Lázár, J. G. Ángyán, M. Hollósi, P. Huszthy and P. R. Surján, Chirality, 2002, 14, 377-385.

4 Y. Tang and A. E. Cohen, Phys. Rev. Lett., 2010, 104, 163901. 5 J. R. Rouxel, V. Y. Chernyak and S. Mukamel, Chem. Sci., 2016, 7, 6824-6831.

$6 \mathrm{~J}$. Goulon, A. Rogalev and C. Brouder, in Comprehensive Chiroptical Spectroscopy, Volume 1, Instrumentation, Methodologies, and Theoretical Simulations, ed. N. Berova, P. L. Polavarapu, K. Nakanishi and R. W. Woody, John Wiley \& Sons, Inc., 2012, ch. 17, pp. 457-491.

7 G. van der Laan and A. I. Figueroa, Coord. Chem. Rev., 2014, 277-278, 95-129. 
8 A. Rogalev, K. Ollefs and F. Wilhelm, in X-Ray Absorption and $X$-Ray Emission Spectroscopy: Theory and Applications, ed. J. A. Van Bokhoven and C. Lamberti, Wiley-Blackwell, 2016, ch. 22, pp. 671-694.

9 L. D. Barron, Molecular Light Scattering and Optical Activity, Cambridge University Press, New York, 2nd edn, 2009.

10 J. Stöhr, Y. Wu, B. Hermsmeier, M. Samant, G. Harp, S. Koranda, D. Dunham and B. Tonner, Science, 1993, 259, 658.

11 Y. Ding, Z. Huang, D. Ratner, P. Bucksbaum and H. Merdji, Phys. Rev. Spec. Top.-Accel. Beams, 2009, 12, 060703.

12 M. Chini, K. Zhao and Z. Chang, Nat. Photonics, 2014, 8, 178.

13 J. D. Biggs, Y. Zhang, D. Healion and S. Mukamel, J. Chem. Phys., 2012, 136, 174117.

14 Y. Zhang, J. D. Biggss and S. Mukamel, J. Mod. Opt., 2014, 61, 558-567.

15 S.-K. Son, H. N. Chapman and R. Santra, J. Phys. B: At., Mol. Opt. Phys., 2013, 46, 164015.

16 K. Tamasaku, E. Shigemasa, Y. Inubushi, T. Katayama, K. Sawada, H. Yumoto, H. Ohashi, H. Mimura, M. Yabashi, K. Yamauchi and T. Ishikawa, Nat. Photonics, 2014, 8, 313.

17 S. Ghimire, A. D. DiChiara, E. Sistrunk, P. Agostini, L. F. DiMauro and D. A. Reis, Nat. Phys., 2011, 7, 138-141.

18 C. Hernández-García, C. G. Durfee, D. D. Hickstein, T. Popmintchev, A. Meier, M. M. Murnane, H. C. Kapteyn, I. J. Sola, A. Jaron-Becker and A. Becker, Phys. Rev. A, 2016, 93, 043855.

19 P. Elleaume, J. Synchrotron Radiat., 1994, 1, 19-26.

20 S. Sasaki, Nucl. Instrum. Methods Phys. Res., Sect. A, 1994, 347, 83-86.

21 J. Goulon, C. Goulon-Ginet, A. Rogalev, V. Gotte, C. Malgrange, C. Brouder and C. R. Natoli, J. Chem. Phys., 1998, 108, 6394-6403.

22 L. Alagna, T. Prosperi, S. Turchini, J. Goulon, A. Rogalev, C. Goulon-Ginet, C. R. Natoli, R. D. Peacock and B. Stewart, Phys. Rev. Lett., 1998, 80, 4799-4802.

23 B. Stewart, R. D. Peacock, L. Alagna, T. Prosperi, S. Turchini, J. Goulon, A. Rogalev and C. Goulon-Ginet, J. Am. Chem. Soc., 1999, 121, 10233-10234.

24 D. J. Higley, K. Hirsch, G. L. Dakovski, E. Jal, E. Yuan, T. Liu, A. A. Lutman, J. P. MacArthur, E. Arenholz, Z. Chen, et al., Rev. Sci. Instrum., 2016, 87, 033110.

25 T. Fan, P. Grychtol, R. Knut, C. Hernández-García, D. D. Hickstein, D. Zusin, C. Gentry, F. J. Dollar, C. A. Mancuso, C. W. Hogle, O. Kfir, D. Legut, K. Carva, J. L. Ellis, K. M. Dorney, C. Chen, O. G. Shpyrko, E. E. Fullerton, O. Cohen, P. M. Oppeneer, D. B. MiloÅąeviäĞ, A. Becker, A. A. JaroÅĎ-Becker, T. Popmintchev, M. M. Murnane and H. C. Kapteyn, Proc. Natl. Acad. Sci. U. S. A., 2015, 112, 14206-14211.

26 S. Turchini, N. Zema, S. Zennaro, L. Alagna, B. Stewart, R. D. Peacock and T. Prosperi, J. Am. Chem. Soc., 2004, 126, 4532-4533.

27 M. Tanaka, K. Nakagawa, A. Agui, K. Fujii and A. Yokoya, Phys. Scr., 2005, 115, 873-876.
28 M. Pecul and W. Dzwolak in Optical Spectroscopy and Computational Methods in Biology and Medicine, ed. M. Baranska, Springer Nature, 2014, pp. 161-177.

29 H. Rhee, Y.-G. June, J.-S. Lee, K.-K. Lee, J.-H. Ha, Z. H. Kim, S.-J. Jeon and M. Cho, Nature, 2009, 458, 310-313.

30 E. Runge and E. K. U. Gross, Phys. Rev. Lett., 1984, 52, 9971000.

31 C. A. Ullrich, Time-Dependent Density-Functional Theory: Concepts and Applications, Oxford University Press, New York, 2012.

32 M. Stener, G. Fronzoni and M. de Simone, Chem. Phys. Lett., 2003, 373, 115-123.

33 N. A. Besley and A. Noble, J. Phys. Chem. C, 2007, 111, 33333340 .

34 S. DeBeer-George, T. Petrenko and F. Neese, Inorg. Chim. Acta, 2008, 361, 965-972.

35 W. Liang, S. A. Fischer, M. J. Frisch and X. Li, J. Chem. Theory Comput., 2011, 7, 3540-3547.

36 K. Lopata, B. E. V. Kuiken, M. Khalil and N. Govind, J. Chem. Theory Comput., 2012, 8, 3284-3292.

37 Y. Zhang, J. D. Biggs, D. Healion, N. Govind and S. Mukamel, J. Chem. Phys., 2012, 137, 194306.

38 D. P. Craig and T. Thirunamachandran in Molecular Quantum Electrodynamics, Dover Publications, 1998, ch. 8, pp. 188-189.

39 J. Autschbach, Chirality, 2009, 21, E116-E152.

40 I. Eom, S.-H. Ahn, H. Rhee and M. Cho, Phys. Rev. Lett., 2012, 108, 103901.

41 A. Gonis and W. H. Butler, Multiple Scattering in Solids, Springer New York, 2000.

42 J. J. Rehr, J. J. Kas, F. D. Vila, M. P. Prange and K. Jorissen, Phys. Chem. Chem. Phys., 2010, 12, 5503-5513.

43 O. Bunău and Y. Joly, J. Phys.: Condens. Matter, 2009, 21, 345501.

44 V. Kimberg and N. Kosugi, J. Chem. Phys., 2007, 126, 245101.

45 W. J. Hunt and W. A. Goddard, Chem. Phys. Lett., 1969, 3, 414-418.

46 H. Ågren, V. Carravetta, O. Vahtras and L. G. Pettersson, Chem. Phys. Lett., 1994, 222, 75-81.

47 H. Ågren, V. Carravetta, O. Vahtras and L. G. M. Pettersson, Theor. Chem. Acc., 1997, 97, 14-40.

48 P. L. Altick and A. E. Glassgold, Phys. Rev., 1964, 133, A632A646.

49 M. Stener, A. Lisini and P. Decleva, Chem. Phys., 1995, 191, 141-154.

50 L. Triguero, L. G. M. Pettersson and H. Ågren, Phys. Rev. B: Condens. Matter Mater. Phys., 1998, 58, 8097-8110.

51 O. Takahashi, M. Kimoto and L. G. Pettersson, Chem. Phys., 2015, 450-451, 109-114.

52 V. Carravetta, O. Plachkevytch, O. Vahtras and H. Ågren, Chem. Phys. Lett., 1997, 275, 70-78.

53 O. Vahtras, H. Ågren and V. Carravetta, J. Phys. B: At., Mol. Opt. Phys., 1997, 30, 1493-1501.

54 O. Plashkevych, V. Carravetta, O. Vahtras and H. Ågren, Chem. Phys., 1998, 232, 49-62. 
55 L. Alagna, S. D. Fonzo, T. Prosperi, S. Turchini, P. Lazzeretti, M. Malagoli, R. Zanasi, C. Natoli and P. Stephens, Chem. Phys. Lett., 1994, 223, 402-410.

56 P. Norman, D. M. Bishop, H. J. A. Jensen and J. Oddershede, J. Chem. Phys., 2001, 115, 10323.

57 P. Norman, D. M. Bishop, H. J. A. Jensen and J. Oddershede, J. Chem. Phys., 2005, 123, 194103.

58 U. Ekström, P. Norman, V. Carravetta and H. Ågren, Phys. Rev. Lett., 2006, 97, 143001.

59 A. Jiemchooroj, U. Ekström and P. Norman, J. Chem. Phys., 2007, 127, 165104.

60 A. Jiemchooroj and P. Norman, J. Chem. Phys., 2008, 128, 234304.

61 S. Villaume and P. Norman, Chirality, 2009, 21, E13-E19.

62 J. Kauczor and P. Norman, J. Chem. Theory Comput., 2014, 10, 2449-2455.

63 L. Jensen, J. Autschbach and G. C. Schatz, J. Chem. Phys., 2005, 122, 224115.

64 J. Autschbach, Comput. Lett., 2007, 3, 131-150.

65 K. Yabana and G. F. Bertsch, Phys. Rev. A, 1999, 60, 12711279.

66 K. Lopata and N. Govind, J. Chem. Theory Comput., 2011, 7, 1344-1355.

67 S. Tussupbayev, N. Govind, K. Lopata and C. J. Cramer, J. Chem. Theory Comput., 2015, 11, 1102-1109.

68 J. J. Goings and X. Li, J. Chem. Phys., 2016, 144, 234102.

69 J. Autschbach and T. Ziegler, J. Chem. Phys., 2002, 116, 891896.

70 J. Autschbach, T. Ziegler, S. J. A. van Gisbergen and E. J. Baerends, J. Chem. Phys., 2002, 116, 6930-6940.

71 J. Autschbach, ChemPhysChem, 2011, 12, 3224-3235.
72 M. Valiev, E. Bylaska, N. Govind, K. Kowalski, T. Straatsma, H. V. Dam, D. Wang, J. Nieplocha, E. Apra, T. Windus and W. de Jong, Comput. Phys. Commun., 2010, 181, 1477-1489.

73 I. Tamm, J. Phys., 1945, 9, 449-460.

74 S. M. Dancoff, Phys. Rev., 1950, 78, 382-385.

75 S. Hirata and M. Head-Gordon, Chem. Phys. Lett., 1999, 314, 291-299.

76 A. D. Becke, J. Chem. Phys., 1993, 98, 5648-5652.

77 P. J. Stephens, F. J. Devlin, C. F. Chabalowski and M. J. Frisch, J. Phys. Chem., 1994, 98, 11623-11627.

78 T. H. Dunning, J. Chem. Phys., 1989, 90, 1007-1023.

79 D. E. Woon and T. H. Dunning, J. Chem. Phys., 1993, 98, 1358-1371.

80 A. K. Wilson, D. E. Woon, K. A. Peterson and T. H. Dunning, J. Chem. Phys., 1999, 110, 7667-7676.

81 R. Krishnan, J. S. Binkley, R. Seeger and J. A. Pople, J. Chem. Phys., 1980, 72, 650-654.

82 A. D. McLean and G. S. Chandler, J. Chem. Phys., 1980, 72, 5639-5648.

83 J.-M. Chen, R. Klauser, S.-C. Yang and C.-R. Wen, Chem. Phys. Lett., 1995, 246, 285-290.

84 T. Fransson, D. Burdakova and P. Norman, Phys. Chem. Chem. Phys., 2016, 18, 13591-13603.

85 G. H. Zschornack, Handbook of X-Ray Data, SprinSpr-Verlag, Berlin, Heidelberg, New York, 2007.

86 S. Tretiak and S. Mukamel, Chem. Rev., 2002, 102, 31713212.

87 C.-F. Chen and Y. Shen, Helicene Chemistry: From Synthesis to Applications, Springer-Verlag, Berlin, Heidelberg, 2017.

88 J. R. Rouxel, M. Kowalewski and S. Mukamel, Struct. Dyn., 2017, 4, 044006. 